Clinical Studies

\title{
On the diagnostic discrimination ability of mouthrinse and salivary aMMP-8 point-of-care testing regarding periodontal health

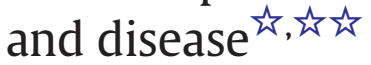

\author{
Ismo T. Räisänen ${ }^{\mathrm{a}, *}$, Anna Maria Heikkinen ${ }^{\mathrm{a}}$, Solomon O. Nwhator ${ }^{\mathrm{b}}$, Kehinde A. Umeizudike ${ }^{\mathrm{c}}$, \\ Taina Tervahartiala ${ }^{\mathrm{a}}$, Timo Sorsa ${ }^{\mathrm{a}, \mathrm{d}}$ \\ a Department of Oral and Maxillofacial Diseases, Head and Neck Center, University of Helsinki and Helsinki University Hospital, PO Box 63, (Haartmaninkatu 8), FI-00014, Helsinki, Finland \\ ${ }^{\mathrm{b}}$ Department of Preventive E' Community Dentistry, Faculty of Dentistry, College of Health Sciences, Obafemi Awolowo University, Ile-Ife, Nigeria \\ c Department of Preventive Dentistry, Faculty of Dental Sciences, College of Medicine, University of Lagos, Idi-araba, Lagos, Nigeria \\ d Division of Periodontology, Department of Dental Medicine, Karolinska Institutet, Stockholm SE-171 77, Sweden
}

\section{A R T I C L E I N F O}

\section{Article history:}

Received 1 June 2019

Received in revised form 18 July 2019

Accepted 20 July 2019

Available online 26 July 2019

Keywords:

Biomarkers

Periodontitis

Matrix metalloproteinase 8

Point-of-care testing

Saliva

Mouthrinse

\begin{abstract}
A B S T R A C T
This study investigated the diagnostic utility of mouthrinse and saliva in aMMP-8 measurements to analyze patients' risk for active periodontal tissue destruction and progression of periodontal disease among 47 adolescents. Results show that measurements from mouthrinse produce better discrimination and should be used instead of saliva measurements.
\end{abstract}

(C) 2019 Elsevier Inc. All rights reserved.
Oral fluids (saliva, mouthrinse, gingival crevicular fluid [GCF], and peri-implant sulcular fluid [PISF]) provide a matrix for periodontitis and dental peri-implant biomarker diagnostics as recently studied by Schmalz et al. (2019). In periodontitis and peri-implantitis, elevated levels of neutrophil collagenase or collagenase-2, especially in active/ activated form, reflect, predict, and monitor the diagnosis, course, and treatment of these diseases. Oral fluid active matrix metalloproteinase-8 (aMMP-8)/neutrophil collagenase is a potential point-of-care $(\mathrm{PoC}) /$ chairside biomarker for these diseases, and commercially available aMMP-8 PoC tests have been developed and are in

\footnotetext{
th Conflict of interestThis work was supported by grants from the University of Helsinki Research Foundation (TYH 2016251, TYH 2017251, TYH 2018229, TYH 2019314 Y1014SL017, Y1014SL018, Y1014SULE1), Helsinki, Finland, and the Karolinska Institutet, Stockholm, Sweden, and Helsingin Seudun Hammaslääkärit r.y.. (Dentists of Helsinki Region Association), Finland. Timo Sorsa is an inventor of US-patents 5652223, 5736341, 5866432, 6143476, 20170023571A1 (granted 6.6.2019), and WO 2018/060553 A1 (granted 31.5.2018). Other authors report no conflicts of interest related to this study.

is is Ethical approvalThis study was approved by the Ethical Committee of Helsinki University Central Hospital, Helsinki, Finland (Diary number 260/13/03/00/13, 17.12.2013). The study was conducted according to the principles of the Declaration of Helsinki (2008). Participants provided a written informed consent in this study.

* Corresponding author.

E-mail address: ismo.raisanen@helsinki.fi (I.T. Räisänen).
}

use. GCF and PISF have often been sampled at individual sites using filter papers and/or micropipettes (Rathnayake et al. 2017), but there is an alternative way to sample GCF: a wash using a mouthrinse technique (Drouin et al. 1988; Gangbar et al. 1990). This simpler technique utilizing mouthrinse has been developed to collect GCF from periodontitis patients (Gangbar et al. 1990; Nwhator et al. 2014; Heikkinen et al. 2016; Räisänen et al. 2019a). The aim of this note is to provide more accurate assessment of the efficiency of mouthrinse vs. salivary aMMP-8 analysis in discrimination of periodontal health and disease.

Fig. 1 illustrates the ability of aMMP-8 PoC mouthrinse test compared to aMMP-8 IFMA measured from saliva, and it clearly shows how saliva is inferior to mouthrinse in aMMP-8 measurements related to identifying periodontal health and disease. There is a significant association between the aMMP- 8 PoC mouthrinse test result and aMMP-8 IFMA levels in saliva in this sample of adolescents, as has been shown recently by Räisänen et al. (2019b). However, if patient's periodontal health is considered, the distinction of salivary aMMP-8 IFMA measurements is far from perfect between a positive and negative test result. aMMP-8 IFMA concentrations vary between similar maximum and minimum levels independent of the patient's number of sites ( 0 or 1 , and 2 or more) with $\geq 4 \mathrm{~mm}$ periodontal pockets. On the contrary, the aMMP-8 mouthrinse test is very sensitive for at least 2 sites with 


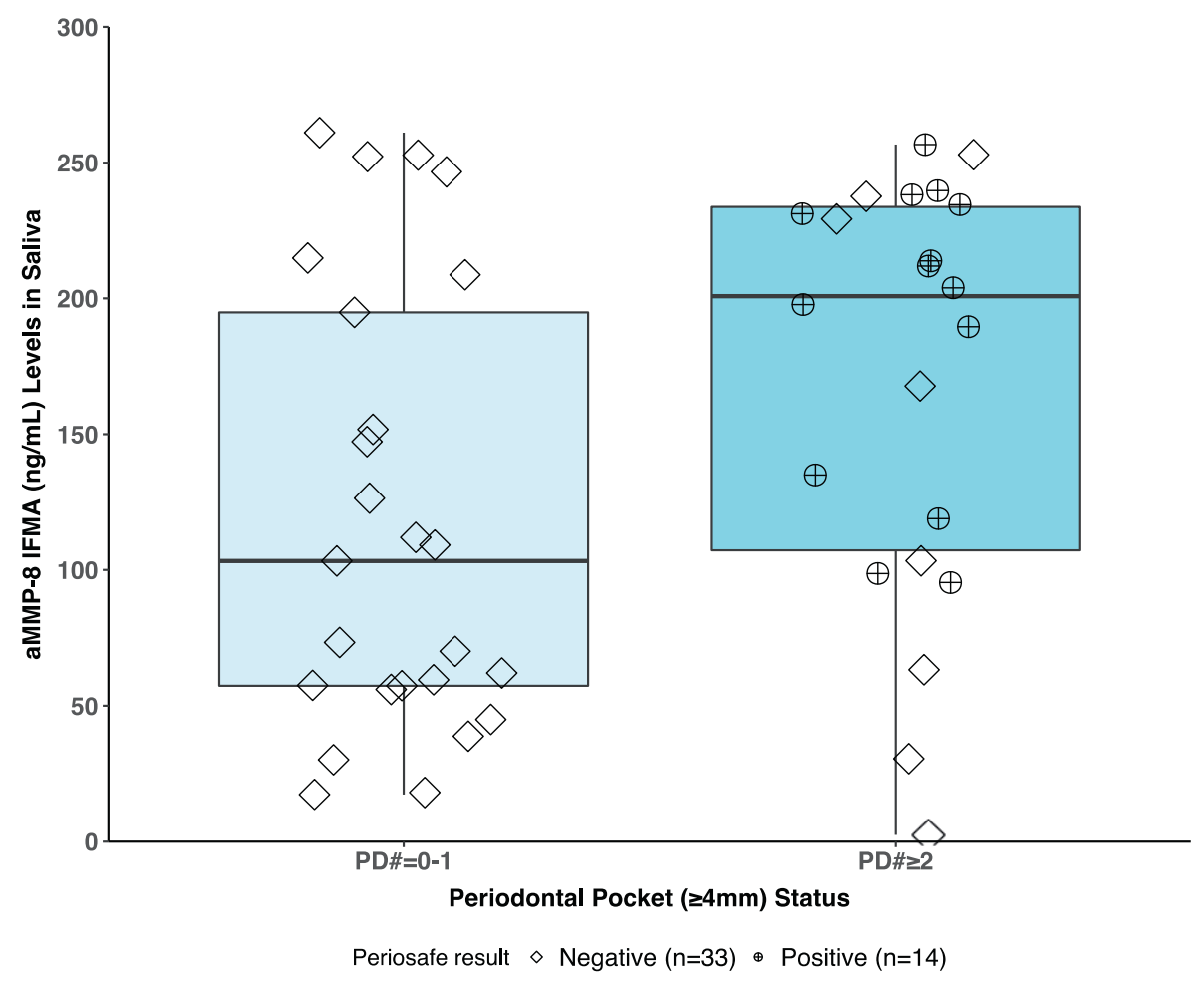

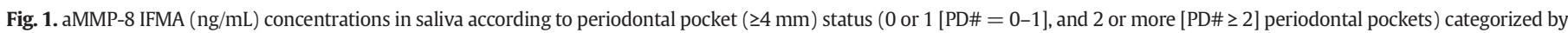
the corresponding aMMP-8 PoC test result measured from oral mouthrinse.

periodontal pockets (Nwhator et al. 2014; Heikkinen et al. 2016; Räisänen et al. 2019a). It is instructive that the sensitivity regarding 2 sites with periodontal pockets of $\geq 4 \mathrm{~mm}$ had been reported in 2014 long before it was mentioned in the latest classification (Tonetti et al. 2018).

As indicated in Fig. 1, aMMP-8 PoC mouthrinse test functions much better and more precisely, which suggests that mouthrinse aMMP- 8 measurements are the optimal way of analyzing patient's risk for active periodontal tissue destruction and progression of periodontal disease. Furthermore, the use of mouthrinse aMMP-8 to measure active collagenases avoids the interference by tissue inhibitors of metalloproteinase (TIMPs) when saliva is used (Drouin et al. 1988; Gangbar et al. 1990).

In accordance with the original findings of Drouin et al. (1988) and Gangbar et al. (1990), as well the recent studies using the aMMP-8 chairside PoC tests (Nwhator et al. 2014; Izadi Borujeni et al. 2015; Heikkinen et al. 2016; Johnson et al. 2016; Sorsa et al. 2016; Heikkinen et al. 2017; Lorenz et al. 2017; Alassiri et al. 2018; Leppilahti et al. 2018; Räisänen et al. 2018; Schmidt et al. 2018; Schmalz et al. 2019; Räisänen et al. 2019a), repeatedly and strongly indicate that aMMP- 8 or active neutrophil collagenase present in mouthrinse is derived from GCF and neutrophils. Regarding saliva, there are multiple other potential sources for aMMP-8 in addition to neutrophils (Rathnayake et al., 2017). Overall, mouthrinse provides calibration and standardization of the GCF and its aMMP-8 biomarker to be quantitatively analyzed (Nwhator et al. 2014; Izadi Borujeni et al. 2015; Heikkinen et al. 2016; Johnson et al. 2016; Sorsa et al. 2016; Heikkinen et al. 2017; Lorenz et al. 2017; Sorsa et al. 2017; Alassiri et al. 2018; Leppilahti et al. 2018; Räisänen et al. 2018; Schmidt et al. 2018; Schmalz et al. 2019; Räisänen et al. 2019a). Additional studies have further shown that aMMP-8, but not total MMP-8, can predict and differentiate between "active" and "inactive" periodontal pockets (Lee et al. 1995; Mancini et al. 1999; Romanelli et al. 1999; Kiili et al. 2002; Sorsa et al. 2006), and standard-of-care treatment (i.e., scaling and root planing) reduces aMMP-8 levels in oral fluid (GCF and mouthrinse) and, at the same time, ceases the clinical progression of the disease (Mäntylä et al.
2003; Leppilahti et al. 2014; Leppilahti et al. 2015; Alassiri et al. 2018). Elevation of aMMP-8 ( $>20 \mathrm{ng} / \mathrm{mL}$ ) by 2 or even 1 active deep site is required for the chairside PoC test to be positive to indicate increased risk for periodontitis and/or its development and progression, thus reflecting the high sensitivity of commercially available aMMP-8 PoC mouthrinse test (Nwhator et al. 2014; Heikkinen et al. 2016; Räisänen et al. 2019a). This was also originally observed for active collagenase activity by Gangbar et al. (1990). Salivary analysis of periodontitis biomarkers is on the other hand less exact and potentially erroneous (Fig. 1). Thus, the aMMP-8 oral fluid test is a mouthrinse-not salivary -test addressing GCF/PISF aMMP-8 in periodontal disease.

\section{References}

Alassiri S, Parnanen P, Rathnayake N, Johannsen G, Heikkinen AM, Lazzara R, et al. The ability of quantitative, specific, and sensitive point-of-care/chair-side oral fluid immunotests for aMMP-8 to detect periodontal and peri-implant diseases. Dis Markers 2018;1306396. https://doi.org/10.1155/2018/1306396.

Drouin L, Overall CM, Sodek J. Identification of matrix metalloendoproteinase inhibitor (TIMP) in human parotid and submandibular saliva: partial purification and characterization. J Periodontal Res 1988;23:370-7.

Gangbar S, Overall CM, McCulloch CA, Sodek J. Identification of polymorphonuclear leukocyte collagenase and gelatinase activities in mouthrinse samples: correlation with periodontal disease activity in adult and juvenile periodontitis. J Periodontal Res 1990;25:257-67.

Heikkinen AM, Nwhator SO, Rathnayake N, Mäntylä P, Vatanen P, Sorsa T. Pilot study on oral health status as assessed by an active matrix metalloproteinase- 8 chairside mouthrinse test in adolescents. J Periodontol 2016;87:36-40. https://doi.org/10. 1902/jop.2015.150377.

Heikkinen AM, Raivisto T, Kettunen K, Kovanen L, Haukka J, Pakbaznejad Esmaeili E, et al Pilot study on the genetic background of an active matrix metalloproteinase- 8 test in Finnish adolescents. J Periodontol 2017;88:464-72. https://doi.org/10.1902/jop.2016. 160441.

Izadi Borujeni S, Mayer M, Eickholz P. Activated matrix metalloproteinase-8 in saliva as diagnostic test for periodontal disease? A case-control study. Med Microbiol Immunol 2015;204:665-72. https://doi.org/10.1007/s00430-015-0413-2.

Johnson N, Ebersole JL, Kryscio RJ, Danaher RJ, Dawson 3rd D, Al-Sabbagh M, et al. Rapid assessment of salivary MMP-8 and periodontal disease using lateral flow immunoassay. Oral Dis 2016;22:681-7. https://doi.org/10.1111/odi.12521.

Kiili M, Cox SW, Chen HY, Wahlgren J, Maisi P, Eley BM, et al. Collagenase-2 (MMP-8) and collagenase-3 (MMP-13) in adult periodontitis: molecular forms and levels in 
gingival crevicular fluid and immunolocalisation in gingival tissue. J Clin Periodontol 2002;29:224-32. https://doi.org/10.1034/j.1600-051x.2002.290308.x.

Lee W, Aitken S, Sodek J, McCulloch CA. Evidence of a direct relationship between neutrophil collagenase activity and periodontal tissue destruction in vivo: role of active enzyme in human periodontitis. J Periodontal Res 1995;30:23-33. https://doi.org/10. 1111/j.1600-0765.1995.tb01249.x.

Leppilahti JM, Kallio MA, Tervahartiala T, Sorsa T, Mäntylä P. Gingival crevicular fluid matrix metalloproteinase-8 levels predict treatment outcome among smokers with chronic periodontitis. J Periodontol 2014:85:250-60. https://doi.org/10.1902/jop.2013.130156.

Leppilahti JM, Sorsa T, Kallio MA, Tervahartiala T, Emingil G, Han B, et al. The utility of gingival crevicular fluid matrix metalloproteinase- 8 response patterns in prediction of site-level clinical treatment outcome. J Periodontol 2015;86:777-87. https://doi. org/10.1902/jop.2015.140421.

Leppilahti JM, Harjunmaa U, Järnstedt J, Mangani C, Hernández M, Tervahartiala T, et al. Diagnosis of newly delivered mothers for periodontitis with a novel oral-rins aMMP-8 point-of-care test in a rural Malawian population. Diagnostics (Basel) 2018;8, E67. https://doi.org/10.3390/diagnostics8030067.

Lorenz K, Keller T, Noack B, Freitag A, Netuschil L, Hoffmann T. Evaluation of a novel point-of-care test for active matrix metalloproteinase-8: agreement between qualitative and quantitative measurements and relation to periodontal inflammation. J Periodontal Res 2017;52:277-84. https://doi.org/10.1111/jre.12392.

Mancini S, Romanelli R, Laschinger CA, Overall CM, Sodek J, McCulloch CA. Assessment of a novel screening test for neutrophil collagenase activity in the diagnosis of periodontal diseases. J Periodontol 1999;70:1292-302. https://doi.org/10.1902/jop.1999.70.11.1292.

Mäntylä P, Stenman M, Kinane DF, Tikanoja S, Luoto H, Salo T, et al. Gingival crevicular fluid collagenase-2 (MMP-8) test stick for chair-side monitoring of periodontitis. J Periodontal Res 2003;38:436-9. https://doi.org/10.1034/j.1600-0765.2003.00677.x.

Nwhator SO, Ayanbadejo PO, Umeizudike KA, Opeodu OI, Agbelusi GA, Olamijulo JA, et al Clinical correlates of a lateral-flow immunoassay oral risk indicator. J Periodonto 2014;85:188-94. https://doi.org/10.1902/jop.2013.130116.

Räisänen IT, Heikkinen AM, Siren E, Tervahartiala T, Gieselmann DR, van der Schoor GJ et al. Point-of-care/chairside aMMP-8 analytics of periodontal diseases' activity and episodic progression. Diagnostics (Basel) 2018;8, E74. https://doi.org/10.3390/ diagnostics8040074

Räisänen IT, Sorsa T, van der Schoor G-J, Tervahartiala T, van der Schoor P, Gieselmann DR, et al. Active matrix metalloproteinase- 8 point-of-care $(\mathrm{PoC}) /$ chairside mouthrinse test vs bleeding on probing in diagnosing subclinical periodontitis in adolescents. Diagnostics (Basel) 2019a;9, E34. https://doi.org/10.3390/diagnostics9010034.

Räisänen IT, Heikkinen AM, Pakbaznejad Esmaeili E, Tervahartiala T, Pajukanta R, Silbereisen A, Bostanci N, Sorsa T. A point-of-care test of active matrix metalloproteinase-8 (aMMP-8) predicts triggering receptor expressed on myeloid cells-1 (TREM-1) levels in saliva. J Periodontol 2019b;1-8. https://doi.org/10.1002/JPER.190132.

Rathnayake N, Gieselmann DR, Heikkinen AM, Tervahartiala T, Sorsa T. Salivary diagnostics-point-of-care diagnostics of MMP-8 in dentistry and medicine. Diagnostics (Basel) 2017;7, E7. https://doi.org/10.3390/diagnostics7010007.

Romanelli R, Mancini S, Laschinger C, Overall CM, Sodek J, McCulloch CA. Activation of neutrophil collagenase in periodontitis. Infect Immun 1999;67:2319-26. [PubMed].

Schmalz G, Hübscher AE, Angermann H, Schmidt J, Schmickler J, Legler TJ, et al. Associations of chairside salivary aMMP-8 findings with periodontal parameters, potentially periodontal pathogenic bacteria and selected blood parameters in systemically healthy adults. Diagn Microbiol Infect Dis 2019(19):S0732-8893. https://doi.org/10. 1016/j.diagmicrobio.2019.05.006. 30094-X.

Schmidt J, Guder U, Kreuz M, Löffler M, Kiess W, Hirsch C, et al. aMMP-8 in correlation to caries and periodontal condition in adolescents-results of the epidemiologic LIFE child study. Clin Oral Investig 2018;22:449-60. https://doi.org/10.1007/s00784017-2132-0.

Sorsa T, Tjäderhane L, Konttinen YT, Lauhio A, Salo T, Lee HM, et al. Matrix metalloproteinases: contribution to pathogenesis, diagnosis and treatment of periodontal inflammation. Ann Med 2006;38:306-21. https://doi.org/10.1080/ 07853890600800103.

Sorsa T, Gürsoy UK, Nwhator S, Hernandez M, Tervahartiala T, Leppilahti J, et al. Analysis of matrix metalloproteinases, especially MMP-8, in gingival crevicular fluid, mouthrinse and saliva for monitoring periodontal diseases. Periodontology 2000 2016;70:142-63. https://doi.org/10.1111/prd.12101.

Sorsa T, Gieselmann D, Arweiler NB, Hernández M. A quantitative point-of-care test for periodontal and dental peri-implant diseases. Nat Rev Dis Primers 2017;3:17069. https://doi.org/10.1038/nrdp.2017.69.

Tonetti MS, Greenwell H, Kornman KS. Staging and grading of periodontitis: framework and proposal of a new classification and case definition. J Periodontol 2018;89 (Suppl. 1):S159-72. https://doi.org/10.1002/JPER.18-0006. 